ORIENTAL JOURNAL OF
ISSN: 0974-6471
June 2017,
COMPUTER SCIENCE \& TECHNOLOGY

\title{
An Optimal Resource Provisioning Algorithm for Cloud Computing Environment
}

\author{
SHIVANGI NIGAM and ABHISHEK BAJPAI \\ Department of Computer Application Shri Ramswaroop \\ Memorial University, Deva Road Lucknow \\ Corresponding author E-mail: shivi.nigam15@gmail.com \\ http://dx.doi.org/10.13005/ojcst/10.02.17
}

(Received: April 04, 2017; Accepted: April 14, 2017)

\begin{abstract}
Resource Provisioning in a Cloud Computing Environment ensures flexible and dynamic access of the cloud resources to the end users. The Multi-Objective Decision Making approach considers assigning priorities to the decision alternatives in the environment. Each alternative represents a cloud resource defined in terms of various characteristics termed as decision criteria. The provisioning objectives refer to the heterogeneous requirements of the cloud users. This research study proposes a Resource Interest Score Evaluation Optimal Resource Provisioning (RISE-ORP) algorithm which uses Analytical Hierarchy Process (AHP) and Ant Colony Optimization (ACO) as a unified MOMD approach to design an optimal resource provisioning system. It uses AHP as a method to rank the cloud resources for provisioning. The ACO is used to examine the cloud resources for which resource traits best satisfy the provisioning. The performance of this approach is analyzed using CloudSim. The experimental results show that our approach offers improvement in the performance of previously used AHP approach for resource provisioning.
\end{abstract}

Keywords: Cloud Computing; Resource Provisioning, Analytical Hierarchy Process; Ant colony Optimization.

\section{INTRODUCTION}

Cloud computing depicts a computing infrastructure, which is accessible to various user applications. The infrastructure incorporates networked computing systems in which various computational applications and resources are available as on demand service to the users ${ }^{1}$. This computing system is a virtualized system that is formed by processing elements that agree to work together by pooling their resources. A distributed network of users requests the Cloud resources via any Cloud Service Provider (CSP). The overall goal of resource provisioning is to allow the applications to utilize computational power, storage, and services. Cloud Resource provisioning requires the CSP to scale and handle user demands seamlessly. To implement a Resource Provisioning System (RPS) as shown in fig 1, a CSP deals with four concerns- Resource Modeling, Discovery, 
Monitoring and Selection². To model the resources in the environment is the first and foremost pillar of the provisioning model of a CSP. The discovery mechanism accounts for the resources ready to be leased for the users. For dynamic provisioning, an RPS needs to be aware of the real time status of the Cloud Resources thus requires a monitor for the system. The selection of resources to be leased to a particular user is based on the status as monitored by the monitoring system.

An efficient resource provisioning system requires a structured technique, for the estimation of the different Cloud resources under various user-application related objectives involved. To help in ranking such multi-objective resources, this research, propose a ranking mechanism with the provisioning of resources as Resource Interest Score Evaluation- Optimal Resource Provisioning (RISE-ORP). The RISE module is based on Analytic Hierarchy Process (AHP) which is one of the most widely used mechanisms for solving problems related to Multi Objective Decision making (MODM). AHP gives a very flexible way for solving such problem and can be adapted to any number of attributes with any number of sub-attributes. The ORP module uses Ant Colony Optimization (ACO) is suitable for the distributed perspective on cloud computing.

The Analytical Hierarchy Process (AHP) is a pair-wise comparison based MODM approach that considers both qualitative and quantitative information and combines them by decomposing ill-structured problems into systematic hierarchies to rank alternatives based on various objectives ${ }^{3,4}$. It provides consistent results for a ranking multiple alternative based on some decision criteria's. It is one of the multi-objective techniques which are suitable for provisioning of cloud computing resources $^{5}$. The AHP has several benefits over other multi-objective decision methods. AHP is an effective means of dealing with the complex decision-making i.e. problems involving decision over a hierarchy of objectives. AHP is also a useful mechanism for checking the consistency of the evaluation measures, which helps a decision-maker to incorporate uniformity in the decision process. AHP computes the priorities for each objective in the hierarchy and thus computes a final score for each alternative. This information gives decision maker a better understanding of the final decision for provisioning. AHP allows inconsistency in judgment because a user is not always consistent, but this inconsistency is allowed only to a certain limit. This limit is checked in AHP by a consistency metric. Thus the AHP results into consistent priorities of the resources.

The real time situation of the cloud computing environment is dynamic and complicated. There are many unexpected large changes occurring in the cloud environment. The provisioning is based only on the user application objectives needs to be improvised for meeting all the above issues of the cloud computing environment. ACO is used to optimize the result of provisioning by considering the characteristics of the cloud environment. Many

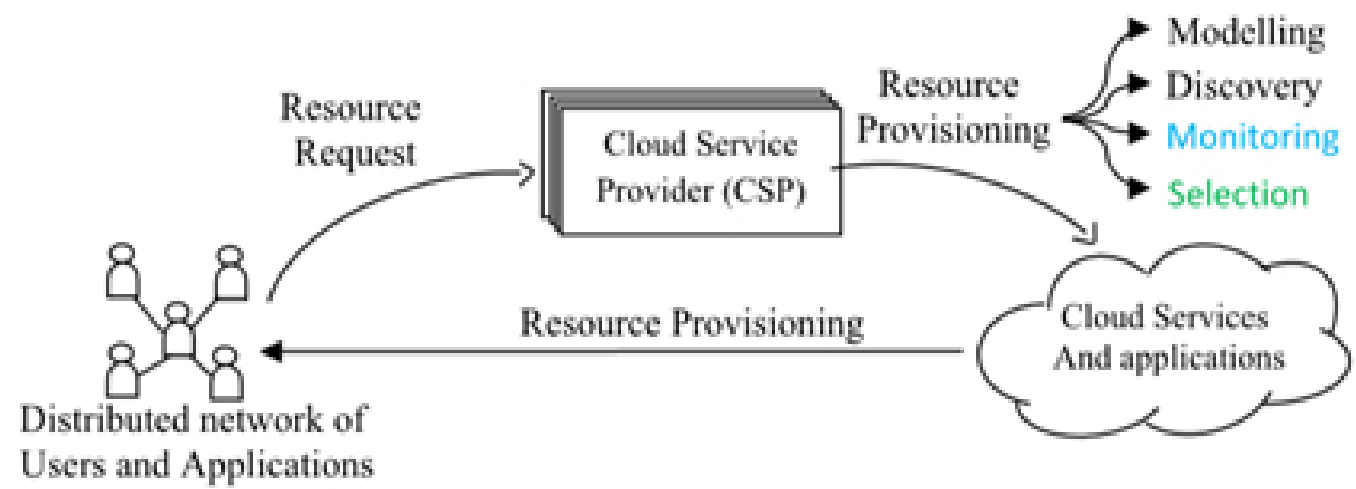

Fig. 1: Resource Provisioning System in Cloud Computing (RPS) 
Artificial intelligence techniques have been used in research for optimizations. Swarm intelligence is an approach to problem solving that takes inspiration from the social behaviors of insects and of other animals. Ant colony optimization (ACO) takes inspiration from the foraging behavior of some ant species. These ants deposit pheromone on the ground in order to mark same favorable path that should be followed by other members of the colony. $\mathrm{ACO}$ is used mainly for handling Optimal Path related problem ${ }^{6,8}$. The problem is to find an optimal path for a given set of alternatives. Considering Cloud Computing, resource provisioning is a dynamic problem as the search space changes by time. Thus, it is crucial that the algorithm be able to adjust the provisioning solutions, following the changes of the target cloud resources. This work incorporates Ant Colony Optimization (ACO) into the AHP for probing the decision of assigning the resource based just on the multi-objectives of the user application. The contents of this paper are as follows: Section 2 presents related works in the concerned area. In Section 3, we introduce the Resource Interest Score Evaluation- Optimal Resource Provisioning (RISE-ORP) model for cloud resource provisioning. It also elaborates the mathematical model and implementation details of the RISE-ORP algorithm. Section 4 presents

Table 1: Literature Details of Resource Provisioning in Cloud Computing

\begin{tabular}{|c|c|c|c|c|}
\hline $\begin{array}{l}\text { Paper Details } \\
\text { Paper }\end{array}$ & $\begin{array}{l}\text { Paper } \\
\text { Subject }\end{array}$ & $\begin{array}{l}\text { source Provisi } \\
\text { Goals } \\
\text { Computational } \\
\text { Resources }\end{array}$ & $\begin{array}{l}\text { Oning } \\
\text { Network } \\
\text { Resources }\end{array}$ & $\begin{array}{c}\text { Energy } \\
\text { Consumption }\end{array}$ \\
\hline $\begin{array}{l}\text { Xiayu et al., } \\
(2010)[7]\end{array}$ & $\begin{array}{c}\text { Ant Colony } \\
\text { Optimization (ACO) }\end{array}$ & $\sqrt{ }$ & $\sqrt{ }$ & \\
\hline $\begin{array}{l}\text { Ergu et. al, } \\
\text { (2011) [5] }\end{array}$ & $\begin{array}{l}\text { Analytical Hierarchy } \\
\text { Process (AHP) }\end{array}$ & $\sqrt{ }$ & & \\
\hline $\begin{array}{l}\text { Lee et al., } \\
(2011)[19]\end{array}$ & $\begin{array}{c}\text { Topology Aware } \\
\text { Resource Allocation (TARA) }\end{array}$ & $\sqrt{ }$ & & \\
\hline $\begin{array}{l}\text { Mohan et al., } \\
(2012)[10]\end{array}$ & $\begin{array}{c}\text { Resource provisioning } \\
\text { Challenges }\end{array}$ & & & \\
\hline $\begin{array}{l}\text { Reddy et al., } \\
(2012)[18]\end{array}$ & NEPHELE & $\sqrt{ }$ & & \\
\hline $\begin{array}{l}\text { Abu et al., } \\
(2013)[11]\end{array}$ & $\begin{array}{l}\text { Resource Provisioning } \\
\text { Network Issues }\end{array}$ & & $\sqrt{ }$ & \\
\hline $\begin{array}{l}\text { Gouda et al., } \\
(2013)[19]\end{array}$ & $\begin{array}{c}\text { Priority Based } \\
\text { Resource Allocation }\end{array}$ & $\sqrt{ }$ & & \\
\hline $\begin{array}{l}\text { Jebalia et al., } \\
(2013)[15]\end{array}$ & $\begin{array}{c}\text { Resource Provisioning } \\
\text { survey of Game } \\
\text { Theoretic Approaches }\end{array}$ & $\sqrt{ }$ & & $\sqrt{ }$ \\
\hline $\begin{array}{l}\text { LeóN et al. } \\
(2013)[14]\end{array}$ & $\begin{array}{l}\text { Energy Savings for } \\
\text { Resource provisioning }\end{array}$ & & & $\sqrt{ }$ \\
\hline Gao, (2014) [8] & Improved ACO & $\sqrt{ }$ & $\sqrt{ }$ & $\sqrt{ }$ \\
\hline $\begin{array}{l}\text { Shu et al. } \\
(2014) z \text { [7] }\end{array}$ & $\begin{array}{c}\text { Resource Provisioning } \\
\text { for Green } \\
\text { Cloud Computing }\end{array}$ & $\sqrt{ }$ & & $\sqrt{ }$ \\
\hline $\begin{array}{l}\text { Singh et. al, } \\
(2014)[21]\end{array}$ & $\begin{array}{c}\text { Multi-Criteria } \\
\text { Preference } \\
\text { Synthesis (MPS) }\end{array}$ & $\sqrt{ }$ & & \\
\hline
\end{tabular}


the analysis of the proposed algorithm and thus produces the corresponding results. This paper uses Cloud-Sim ${ }^{9}$ as the simulation platform and compares the RISE-ORP algorithm with the AHP resource provisioning policy. The simulation experiments on Cloud-Sim to verify the superiority of RISE-ORP algorithm; Section 5 concludes the paper and discusses the direction of future research.

\section{Related work}

Resource Provisioning Techniques in Cloud Computing aims to guarantee that the application requirements are attended correctly ${ }^{2}$. Resource provisioning relies heavily on the strong algorithms for allocating properly CPU, RAM, hard disk and other computational resources to the intended applications. To maintain its scalability, the process of provisioning in Cloud Computing must be dynamic. The authors in ${ }^{10}$ estimated various network resource provisioning strategies and their applications in a Cloud Computing Environment. The authors categorized numerous parameters such as high throughput, maximum efficiency, SLA aware, QoS aware, maximum energy and power consumption etc. This work considered only computational resources. The research work in ${ }^{11}$ considered various network issues and energy related issues that can improve the provisioning results. The authors show.

a complete picture of internal and external challenges related to the Network and Energy consumption issues that can be referenced to for designing a complete Resource Provisioning solution. A resource provisioning for Green Cloud Computing proposed by ${ }^{12,13}$ considers designing low-power computing infrastructure, keeping it an energy-efficient and safe. It allocates resources considering the energy consumption and make

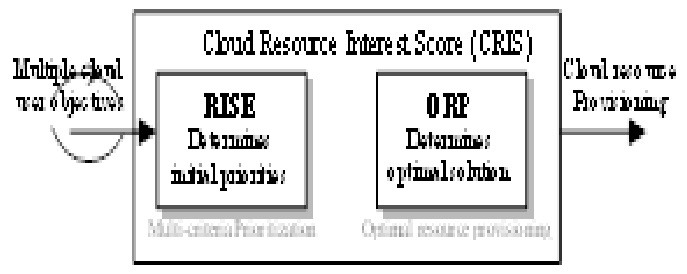

Fig. 2: Optimized MODM Framework for a Resource Provisioning in Cloud Computing span associated with resources. The authors in ${ }^{14}$ investigated the limits of energy savings for the provisioning of data center resources by an algorithm that is efficient in selecting the optimal set of active resources to maximize energy consumption. The problem of energy minimization is modeled as a Stackelberg competition game to compute the upper bound on the energy savings that optimizes both energy consumption and the resource requirements of applications.

A large amount of literature review work has focused on resource provisioning and its challenges. The authors in ${ }^{15}$ give an overview of the resource provisioning models adopted in literature. The authors focus on game theoretic approaches for resource provisioning and suggest a set of criteria so as to examine the performance of the proposed game theory based models with respect to cloud computing needs. The authors also consider addressing the resource provisioning and pricing strategies. A comparison of various bargaining approaches is also presented for resource provisioning in the cloud. Data Envelopment Analysis (DEA $)^{16,17}$ is proposed to evaluate the whole cloud parameters. It can help find out the resource provisioning policy, most suitable between the users and vendors. DEA analyses, resource provisioning problem which includes user's demand, distance, CPU utilization and cost, etc. NEPHELE ${ }^{18}$ was the first to introduce Dynamic provisioning/ de-provisioning of resources in its scheduling and during job execution from a cloud. It considers a number of subtasks with their turnaround time as the algorithm parameters. The main pitfall was High Overload. Priority Based Resource Allocation ${ }^{19}$ decides priority among different user request based on many parameters needed to run the job or task. It results with maximum profit and minimum wastage. The parameters considered are fixed parameters such as time, cost, number of processor request, profit, user, resource assigned, resource availability and resource selection criteria. Topology Aware Resource Allocation (TARA) ${ }^{20}$ gives resource provisioning for Infrastructure-as-a-Service (laaS) based cloud system. Prediction Engine is used to guide provisioning decisions, currently un-optimized for large applications. It focuses on performance of resources. The drawback of this approach is Prediction overhead. 
The genetic techniques inspired by nature are also used to handle the resource provisioning techniques. One of such technique is Ant colony optimization (ACO). ACO was proposed in ${ }^{6}$ to satisfy the needs of Infrastructure-as-a-Service (laaS) in the cloud computing environment. An ACO allocates resources dynamically according to varied user objectives. Parameters are considered according.

To user's requirements. Applying an ACO gives slow Convergence but is guaranteed. Improved ACO as presented in ${ }^{8}$ introduces the factor of energy consumption. It claimed that throughput increased by considering the Energy consumption perspective of a cloud computing environment. Analytical Hierarchy Process has been used for provisioning of resources in a cloud computing environment ${ }^{5}$. This method used Eigen Vector Method for synthesis of all incoming requests for provisioning. There is a pitfall of using Eigen Vector Method that the Inconsistency is increased when the size of the comparison matrix increases. This is a hiccup for a multifarious cloud environment. The research work $\mathrm{in}^{21}$ modified the above technique by using error criteria like Euclidean Distance and Minimum Violation to maintain the consistency in the priorities of the tasks. Euclidean distance is mostly used in optimization problems to compare distances. It works on the principle of straight-line distance in two or three dimensional space. In the cloud environment, this principle not suitable as the actual cloud network requires some generic technique to fit the ill structured cloud. ACO offers a better solution for optimization problems, considering the practical cloud environment.

Resource Interest Score Evaluation- Optimal Resource Provisioning (Rise-Orp) Technique

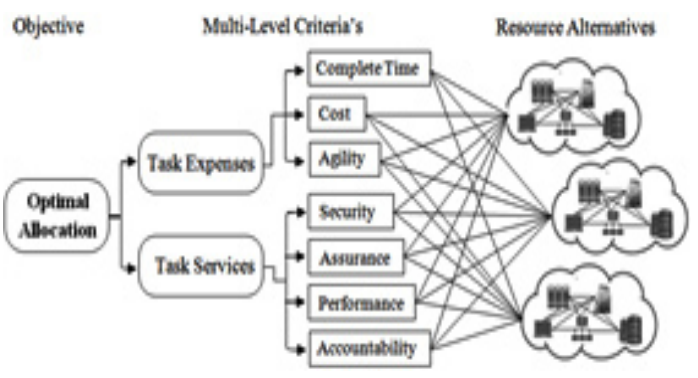

Fig. 3: RISE Hierarchy of Criteria's and Cloud Resource Alternatives
The RISE-ORP model extends the AHP for provisioning in cloud environment as proposed $\mathrm{in}^{5}$. The Resource Provisioning System (RPS) considered for this work is described in section 1. The RPS here is designed so as to model the user oriented and resource oriented characteristics. The proposed work models the provisioning of cloud resources in two modules as Resource Interest score Evaluation (RISE) and Optimal Resource Provisioning (ORP). In the first module, a hierarchy structure is modeled that specifies the interrelation among three kinds of elements, including the overall goal, QoS attributes and their sub-attributes, and alternative services. This module depicts the cloud user oriented characteristics of the RPS. The overall goal of the research is to improve the resource provisioning results based only on the user application objectives, the QoS attributes are the user application requirements for cloud resources and the alternatives are the cloud resources which are to be provisioned to the incoming user request. The AHP is used to provide Cloud Resource Interest Score (CRIS) (weights/priorities) to these alternatives based on the attributes to accomplish the ultimate goal. In the second phase, CRIS of the alternatives is refined for a best optimal solution. This module uses ACO for fulfilling the desired objective. It is designed to model the cloud resource oriented characteristics.

The proposed model tackles the problem of Multi-Objective Decision making for resource provisioning in two modules as shown in Fig 2. RISE helps in relating the alternatives to the goal, and for evaluating Consistent Cloud Resource Interest scores for all alternative cloud resources. The ORP provides optimal solution out of all the consistent options. This work utilizes the advantage of AHP that it has an ability to rank choices in order of their effectiveness in meeting conflicting objectives with

\section{Intensity of Definition Relevance}

Equal importance

Weak importance of one over another Essential or strong importance Demonstrated importance Absolute importance Intermediate values 
efficiently detecting the inconsistent judgments. But as the decision problem is decomposed into a number of subsystems, pair wise comparisons are to be made under each subsystem.

The resource provisioning system proposed here handles the provisioning with the help of the two approaches which handles different aspects of the provisioning system. The RISE deals with the prioritization of alternatives with respect to the attributes as per the RISE hierarchy. The ORP handles the optimization of the priorities on the basis of the cloud computing environmental attributes. The two modules of this work are as:

\section{Resource Interest Score Evaluation (RISE) for Cloud Resources Optimal Resource Provisioning (ORP) for Cloud Computing}

The Resource Interest Score Evaluation for cloud resources provides consistent priorities to the alternatives. The Optimal Resource Provisioning optimizes the Cloud Resource Interest Score (CRIS) provided by the RISE module. The framework accounts, various objectives and assigns consistent weightage to each influencing criteria's. These influencing criteria's vary according to the user requirements. Assigning weightage to the factors ensure that it meets multiple user application objectives. The system will recommend user preference using Analytical Hierarchical Process (AHP) to help in decision making. Fig 3 present a hierarchy of the RISE model as proposed by this work. The RISE hierarchy used in this work is influenced the Services as mentioned in the Service Measurement Index as provided by the Cloud Service Measurement Index Consortium (CSMIC) (SMI Framework, ${ }^{22}$ ). The framework gives a list of QoS attributes for measuring the services provided by a Cloud Service Provider (CSP). The criteria's of the hierarchy are inspired by the services as mentioned in the Cloud SMI framework. The top level of the hierarchy is the ultimate goal of the work. The second and third level represents the criteria's to be met by the RPS. The criteria's are divided into broad categories Task Expenses and Task Services. All the user requests are termed as Task which are to be handled by RPS. The expenses of the cost, a complete time and agility are structured under Task Expenses and Security, Assurance and Performance are under Task
Services. All the alternatives are ranked according to these criteria's. The lowest level of the hierarchy depicts the resource alternatives which are to be mapped to the appropriate alternative. The RISE provides Cloud Resource Interest Score (CRIS) by prioritizing the alternative according to these criteria's. The chosen CRIS will be arranged using the ORP module to find the optimal provisioning.

\section{Resource Interest Score Evaluation (RISE) for Cloud Resources}

Resource Interest Score Evaluation (RISE) uses Analytical Hierarchy Process (AHP) as a principle of hierarchic composition to derive composite priorities of criteria's and alternatives. It computes Cloud Resource Interest Score (CRIS) by AHP method. AHP is a powerful decisionmaking methodology for determining the priorities among different predefined user criteria's. AHP determines the most cost effective solution for resource provisioning. It undergoes an assessment to evaluate each path for the possible uncertainty. Information is decomposed into a hierarchy (Fig 3.) of alternatives and criteria. Information is then synthesized to determine relative ranking of alternatives (Saaty Rating Scale) as shown in Table $2^{4}$.

The matrix is composed by pair wise comparisons, carried out for all factors. Using the pair wise comparisons, the relative importance of one criterion over another can be expressed. For each pair of criteria, the user defines the relative importance of two.

Each priority of alternative is then multiplied by priority of corresponding criterion. Further, adding over all criteria to obtain overall priority of that alternative. It undergoes an assessment to evaluate each alternative to the possible uncertainty. The Fig 4 shows the flowchart of steps followed in this module. This module takes RISE hierarchy criteria's Task Services (TS) and Task Expenses (TE) as inputs.

In order to compute the weights for the different cloud user application criteria's, the M-Learning AHP framework starts creating a pair wise comparison matrix $A$. The matrix $A$ is an $m \times m$ real matrix, where $m$ is the number of evaluation 
criteria considered. Each entry $\mathrm{a}_{\mathrm{j} \mathrm{k}}$ of the matrix $\mathrm{A}$ represents the importance of the $\mathrm{j}^{\mathrm{k}}$ criterion relative to the $k^{\text {th }}$ criterion. If $a_{j k}>1$, then the jth criterion is more important than the $\mathrm{k}^{\text {th }}$ criterion, while if $\mathrm{a}_{\mathrm{jk}}<1$, then the jth criterion is less important than the $\mathrm{k}^{\text {th }}$ criterion. If two criteria have the same importance, then the entry $a_{j k}$ is 1 . The entries $a_{j k}$ and $a_{k j}$ satisfy the following constraint:

$$
\alpha_{j k} \alpha_{k j}
$$

Obviously, $a_{\mathrm{j} j}=1$ for all $\mathrm{j}$. The relative importance between two criteria is measured according to a numerical scale from 1 to 9 called Saaty Rating Scale [3], as shown in Table 2, where it is assumed that the jth criterion is equally or more important than the $\mathrm{k}^{\text {th }}$ criterion.

Step:1. Deriving the relative weight of criteria from the comparison pair wise matrix

At this step, the set of weights (w) that are most consistent with the relativities expressed in comparison matrix (A). The Eigen value method is used to provide the best fit to the judgment recorded in the pair wise comparison matrix $\mathrm{C}$.

$$
A_{w}:=\lambda_{\max } W
$$

$w$ is the weight vector, $\lambda_{\max }$ Is the maximum Eigen value. The resulting matrix $A_{w}$ is the weight matrix, a normalized matrix.

\section{Step:1.Calculate the inconsistency ratio}

When many pair wise comparisons are performed, some inconsistencies may typically arise. The AHP incorporates an effective technique for checking the consistency of the evaluations made by the decision maker when building each of the pair wise comparison matrices involved in the process.

Consistency Index CI $:=\frac{\lambda_{\operatorname{mx}}-\pi}{\pi-1}$

$$
\text { Consistency Ratio } \mathrm{CR}:=\frac{a}{\mathrm{FI}}
$$

Where $n$ is the order of Matrix $A, R I$ is the random index. $R$ I is generated by reciprocal matrix using scale $1 / 9,1 / 8 \ldots 1 \ldots, 8,9$ and get the random consistency index to see if it is about $10 \%$ or less.

If $\mathrm{CR}$ is less than $10 \%$ or 0.10 , then the matrix can be considered as having an acceptable consistency.

Step:2. Computing the rank of each alternative based on the derived relative weight.

The weighted sum method is applied for calculating overall priorities. Weights are assigned to all tasks and resources based on user defined criteria's. The Cloud Resource Interest Score (CRIS) of each optimal alternative is calculated. The best alternative will be selected.

$\mathrm{tj}(\kappa)$ is the attractiveness of the move and is computed by heuristic information indicating a prior

\section{Optimal Resource provisioning in Cloud Computing}

Optimal provisioning of cloud resources, utilizes Ant Colony Optimization (ACO) as a heuristic algorithm for providing the best resource alternative given its priority for a user application. The authors $\mathrm{in}^{6}$ proposed the use of ACO to solve the resource provisioning for cloud computing. It is inspired by the behavior of real ant colonies. The herd of ants works by producing Pheromones. The pheromone is a chemical which the ant leaves on the path they travel. The other ants are able to detect these pheromones and are intended to follow a path with more pheromone content. In the perspective of cloud environment, ORP allots the computational

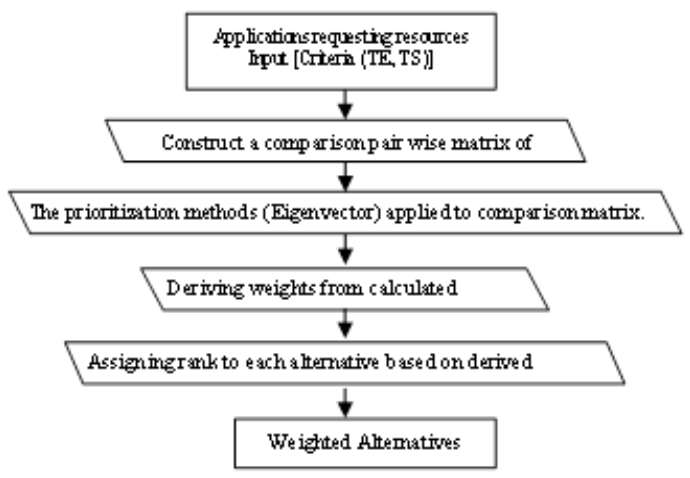

Fig. 4: Resource Interest Score Evaluation (RISE) for prioritization of Cloud Users 
resources to the tasks based on some criteria. If it could be applied in the cloud computing resources provisioning, it can solve many issues such as the difficulty in the prediction of real-time dynamic change of the cloud environment for resource provisioning. The Steps followed by this module are discussed in detail as undergoes.

\section{Step:1. Pheromone Update}

The dynamic behavior of cloud may cause pheromones to misleading ants, which results to a local optimal solution. Thus, an updating timing of the pheromones is selected at the same time when the new alternative is assigned to the compute nodes, because the new alternative will occupy some resources of the node, which will lead to the decrease of the node performance. The initial values are set according to the CRIS of the alternatives. The updating is done according to formula $(4)^{80}$.

$$
T_{j}(j)=(1-\rho)^{\star} T j(j)
$$

- $T_{i}(j)$ is pheromones value of the $i$ resource in $j$ cycles, $0<\mathrm{i}<\mathrm{m}$,

- $\rho$ is the residual coefficient of pheromones,

- $1-\rho$ is the evaporation coefficient of pheromones.

\section{Step:1. Next Hop Calculation:}

The formula in the classical ant colony algorithm, at time $\mathrm{t}$, the probability of the ant $\mathrm{k}$ heading to next point $\mathrm{j}$ is:

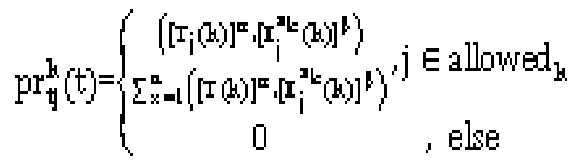

- $\operatorname{prk}_{i j}(t)$ is the probability that the ants choosing the current point, and $a$ and $B$ are the relative weights of pheromones and resource processing speed predictive values.

- $t(k)$ desirability of that move. The CRIS of the alternatives represent the initial desirability of the initial move. It is then updated according to formula (4). ?allowed? $\mathrm{k}$ is the set of all alternative $s$ to be provisioned.

- $E_{i}^{\alpha k}(k)$ is the pheromone trail level of the move, indicating how profitable it would be to make this move. It's the measure of cloud resource for the resource $\mathrm{j}$ for the kth ant.

- $\quad x$ is the set of remaining feasible nodes. Its accounts for all the alternatives to be provisioned.

After initializing the pheromone trails and control parameters, a main loop is repeated until the stopping criterion is met. The stopping criterion for this module is the minimum execution time of the overall execution.

The ORP module finds the best provisioning solution so as the global execution time for the cloud resources is the minimum. In the main loop, ants construct feasible solutions according to the cloud resource parameters and update the associated pheromone trails. At each execution step, ants compute a set of possible moves and select the best one (according to probabilistic rules defined above) to carry out the rest of the tour. The transition probability is based on the heuristic information and the pheromone trail level of the move. The higher the value of the pheromone, the more profitable it is to select this move and resume the search. The module gives the optimal provisioning solution. Fig 5 shows the steps of this module.

\section{Implementation Model}

This section presents the implemented architecture of RISE-ORP model for resource provisioning in cloud computing. The details of RISE hierarchy and Cloud-Sim framework are discussed and the simulation results are enlisted and analyzed.

\section{Rise Hierarchy}

AHP provides a structured technique to provide a consistent ranking to the User alternatives based on the hierarchy. The RISE-ORP model is based solely on the hierarchical attributes chosen for AHP. These attributes are chosen such that they depict the user requirements. These attributes are then arranged in the RISE hierarchy.

The authors in ${ }^{5}$ ranked the alternative based on network bandwidth, task costs and reliability. The hierarchical attributes used for this work are defined in the above section in Fig. 3. The details of these attributes as assumed by this 


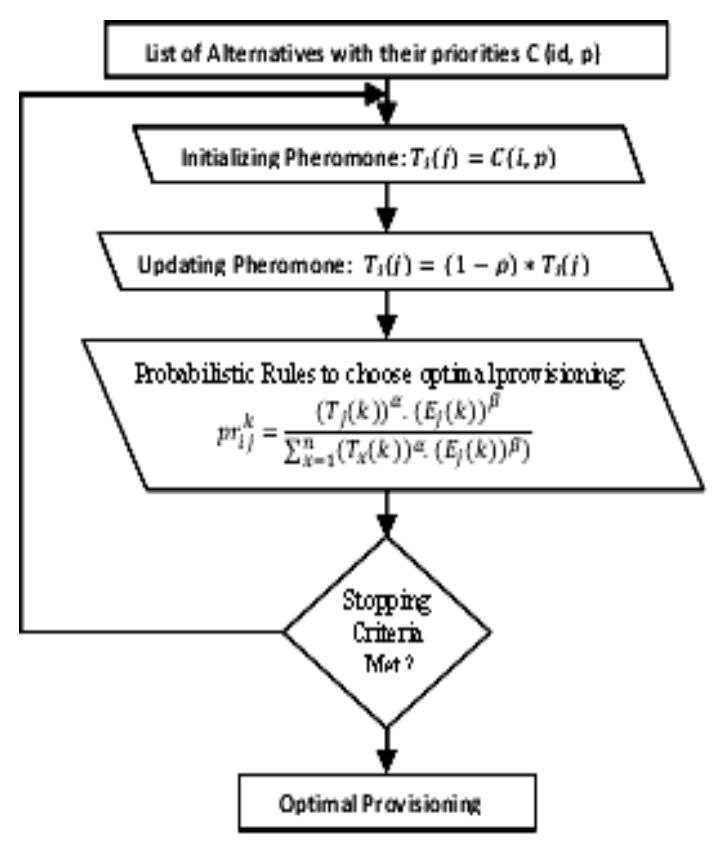

Fig. 5: Optimal Resource Provisioning (ORP) Algorithm for Cloud Computing work are enlisted in Table 3. The assumptions are made to represent user requirements. The weights decide the relative priority of the respective attribute. Based on these details, the AHP is used to compute the RISE matrix resulting in the CRIS for every alternative.

The RISE module represents the alternatives with respect to the hierarchical attribute weights in the pair wise matrix and then calculating the final priorities (CRIS) by removing the inconsistent elements from the pair wise matrix. The inconsistency of the elements may result in unreliable results.

Table 3: RISE hierarchy Details

\begin{tabular}{lc}
\hline Alternative Expenses (0.4) & Cost (0.4) \\
& Completion Time (0.2) \\
& Agility (0.4) \\
Alternative Services (0.6) & Performance (0.5) \\
& Assurance (0.2) \\
& Security (0.3) \\
\hline
\end{tabular}

Table 4: CloudSim Parameters

\begin{tabular}{|c|c|c|c|}
\hline \multirow[b]{2}{*}{ Parameter } & \multicolumn{2}{|c|}{ CloudSim parameters } & \multirow[b]{3}{*}{$\begin{array}{l}\text { Instruction Length } \\
\quad \text { (In Bytes) }\end{array}$} \\
\hline & $\begin{array}{l}\text { Number of } \\
\text { instances }\end{array}$ & $\begin{array}{c}\text { Parameter } \\
\text { details }\end{array}$ & \\
\hline Alternatives & 10 & Alternative Id & \\
\hline & & 0 & 54000 \\
\hline & & 1 & 48000 \\
\hline & & 2 & 80000 \\
\hline & & 3 & 72000 \\
\hline & & 4 & 65000 \\
\hline & & 5 & 78000 \\
\hline & & 6 & 59000 \\
\hline & & 7 & 40000 \\
\hline & & 8 & 30000 \\
\hline & & 9 & 35000 \\
\hline \multirow[t]{5}{*}{ Virtual Machines(VM) } & 2 per $\mathrm{PM}$ & VM Id & MIPS \\
\hline & & 0 & 512 \\
\hline & & 1 & 1024 \\
\hline & & 2 & 128 \\
\hline & & 3 & 256 \\
\hline Physical machines(PM) & 02 & - & \\
\hline Data Centre & 01 & - & \\
\hline CPU Time of Alternatives & $1000 \mathrm{~ms}$ & - & \\
\hline
\end{tabular}


Table 5: CRIS for Alternatives

\begin{tabular}{|c|c|c|c|c|c|c|c|c|c|c|}
\hline C_Id & $\mathrm{CO}$ & C1 & C2 & C3 & C4 & C5 & C6 & C7 & C8 & C9 \\
\hline $\begin{array}{l}\text { RIS } \\
\text { Table }\end{array}$ & 0.520 & 0.485 & 0.461 & 0.551 & 0.675 & 0.516 & 0.786 & 0.727 & 0.637 & 0.642 \\
\hline
\end{tabular}

\section{CloudSim Framework and Optimization of CRIS}

The proposed work is implemented in CloudSim framework. CloudSim is a discrete event based simulator. Every event represents a change in overall system status. Whenever an event occurs, event driven simulation engine processes the events and updates the system status ${ }^{9}$. The simulation involves abstracting cloud computing as a collection of resources which are represented by datacenters

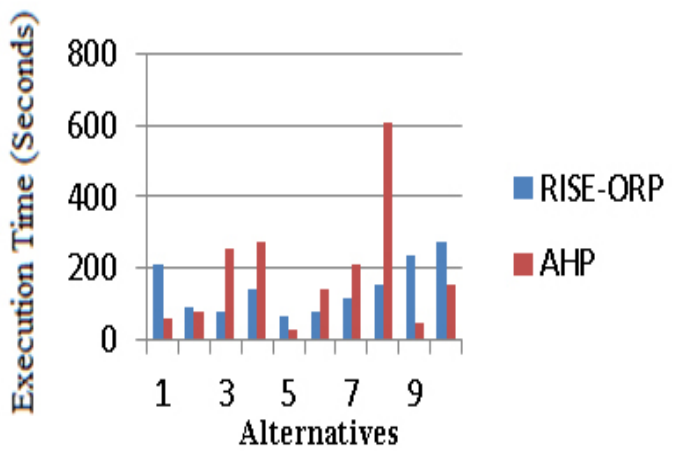

Fig. 6: Comparison of execution times for various Alternatives

Table 6: Execution times for various Alternatives

\begin{tabular}{lcc}
\hline $\begin{array}{l}\text { Alternative } \\
\text { Id }\end{array}$ & $\begin{array}{l}\text { VM } \\
\text { Id }\end{array}$ & $\begin{array}{c}\text { RISE_ORP } \\
\text { Execution Time }\end{array}$ \\
\hline 1 & 3 & 210.94 \\
2 & 0 & 93.75 \\
3 & 1 & 78.13 \\
4 & 0 & 140.63 \\
5 & 1 & 63.48 \\
6 & 1 & 76.17 \\
7 & 0 & 115.23 \\
8 & 3 & 156.25 \\
9 & 2 & 234.37 \\
10 & 2 & 273.44 \\
\hline
\end{tabular}

consisting of a large number of physical machines. These physical machines can have multiple virtual machines. Applications are submitted to the virtual machines. To completely model the cloud computing environment, different components like Datacenter, Physical Machine and VM provisioning are created. The details of modeling CloudSim resources are presented in Table 3.

The CloudSim parameters considered for a Cloud Computing Environment guide the working of the ORP module. The ORP module optimizes the CRIS as obtained by the RISE module. The CloudSim manager handles the ORP module. The management is done by a broker, which handles the communication between the alternative (task) and the datacenter. The Datacenter broker acts on behalf of the user. The Datacenter Broker is responsible for the submission of the alternatives to virtual machines. CloudSim implements simple provisioning policy checking for available Processing Elements (PEs). It supports two-stage commit of

Table 7: Comparison of RISE_ ORP and AHP Execution times

\begin{tabular}{lcc}
\hline Alternative Id & $\begin{array}{c}\text { RISE_ORP } \\
\text { Execution Time }\end{array}$ & $\begin{array}{c}\text { AHP } \\
\text { Execution Time }\end{array}$ \\
\hline 1 & 210.94 & 57.62 \\
2 & 93.75 & 78.13 \\
3 & 78.13 & 253.91 \\
4 & 140.63 & 273.44 \\
5 & 63.48 & 29.30 \\
6 & 76.17 & 140.63 \\
7 & 115.23 & 210.94 \\
8 & 156.25 & 609.38 \\
9 & 234.37 & 46.88 \\
10 & 273.44 & 156.25 \\
Global & 1442.38 & 1856.45 \\
Execution Time & & \\
\hline
\end{tabular}


reservation of hosts: first, reserve the host and, once committed by the user, it is effectively allocated to he/she.

The parameters of the CloudSimparameters are set as in Table 4. The CloudSim simulator was configured with a datacenter composed of a two physical machines - or host in CloudSim terminology - with the same characteristics as the real machine where the experiments were performed. Each job or alternative as submitted by users is called alternative by CloudSim. There Instruction Length is the number of instructions to be executed by an alternative. Moreover, PEs is the number of processing elements (cores) required to perform each individual alternative. Input size and Output size are the input file size and output file size in bytes, $300 \mathrm{~B}$ respectively. Here, processing power is expressed in MIPS (Million Instructions per Second). Once configured, we checked that the execution times obtained by the simulation coincided or were close to the expected result.

The execution times are defined as the time taken by an alternative to use the cloud resources. This work simulates a data center in Cloud-Sim and creates four virtual machines and ten alternatives. The virtual machines are labeled from VMO to VM3 and cloudless are labeled from C0 to C9. The values of these parameters are assumed for the simulation purpose. The virtual machines differ in their MIPS. The various alternatives have various instruction lengths. We assume that other properties of these virtual machines are the same and the bandwidth of links between virtual machines is equal.

\section{Simulation Results}

The analysis consists of simulating the Cloud environment for demonstrating the resource provisioning for cloud resources. The simulation starts by virtualizing a cloud environment in CloudSim. This includes creating the required resources of the cloud network like Data Center, Alternatives (Tasks), Virtual Machine, Physical Machine. The implementation details of the simulated environment are discussed in the previous section. To demonstrate the proposed algorithm, we quantify application performance of Resource Interest Score valuation- Optimal Resource Provisioning
(RISE-ORP) - based resource provisioning. The algorithm consists of two modules. The RISE module computes CRIS for the alternatives by AHP. The RISE works with the attributes and their values listed in the RISE hierarchy in Table 3. The hierarchical attributes cover the alternative oriented parameters and calculate CRIS for the alternatives. The CRIS results for alternatives are mentioned in Table 5. The authors in ${ }^{5}$ mainly focused on the reducing the Inconsistency of the relative pair wise comparison matrix. The authors propose the use of these weights for resource allocation in the cloud environment. They rank the alternatives based on network bandwidth, task costs and reliability. The weights assigned to alternatives decide the priority of the resources to be provisioned. The RISE module results in the consistent weights (CRIS) assigned to the alternatives as in the ${ }^{5}$.

The ORP module deals with the local CRIS provided by the RISE module, resulting in a global optimal solution. The ORP results are calculated with the cloud resource parameters assumed in Table 4. These parameters guide ACO for optimal provisioning of cloud resources. The new weights (CRIS) will be the initial values for the ORP. The ORP runs for 20 iterations and results in the optimal allocation results. The ORP module refines the CRIS for the alternatives and assigns final priorities to the alternatives according to the cloud resource parameters. This module results in execution times of alternatives expressed in seconds. The final provisioning results are as shown in Table 6.

\section{Analysis And Evaluation}

The proposed approach visualizes resource provisioning as a two sided coin. The head of the coin depicts the front end of the resource provisioning problem, i.e. the users. The tail of the coin depicts the back end of the resource provisioning, i.e. the cloud resources. With RISE-ORP we deal with both the sides of the coin. The RISE module focuses the front end issue and ORP module the back end issue. Both the sides are equally important for optimal resource provisioning. This explains the reason to introduce the optimization into the AHP.

The main focus of this approach is to reduce the overall execution times of the alternatives. The 
performance of the proposed approach is compared to the performance of the AHP model as described $\mathrm{in}^{5}$. The resource provisioning in ${ }^{5}$, the resources are provisioned according to the priorities assigned by AHP. RISE-ORP extends the results of AHP to reassign priorities by using ACO. The re-assignment considers the available resources for assigning the resources the best optimal provisioning. The ORP module handles this by computing the cloud resources for the requirements of the alternatives. The provisioning of the alternatives to the resources is based on the calculations of this module.

The comparison results are presented in Table 7. The simulation results show the assignment of $4 \mathrm{VMs}$ among 10 Alternatives with their execution times. The results show increment as well as decrement in the execution times by the RISE-ORP approach. The execution times have increased for the alternatives $\mathrm{C} 3, \mathrm{C} 4, \mathrm{C} 5$ and $\mathrm{C} 6$ as compared to AHP. But the global execution time has been reduced.

The provisioning results of RISE-ORP show improvement of $22.3 \%$ over the provisioning of AHP. The proposed algorithm has been proved to be superior in providing overall optimal solutions to the resource provisioning in a cloud environment. The execution times of the alternative as per the RISE-ORP show a global optimal solution. Though in some cases the execution times have increased, but the overall results have been reduced. Fig. 6 . shows the comparison of execution times for various Alternatives provisioned to the cloud resources by RISE-ORP and AHP respectively.

\section{CONCLUSION}

Priority is an important issue of resource provisioning in cloud environments. In this work, we have analyzed various recent resource provisioning algorithms and categorized them according to their provisioning goals. Considering the heterogeneous cloud environment, we have proposed a priority based optimal provisioning algorithm, RISE-ORP which can be applied in cloud environments. It is best suited for the multicriteria prioritization of the various user demands in the dynamic cloud environment. In this work we have successfully compared our algorithm with a priority based resource provisioning algorithm the AHP and saw that execution time has been improved considering the overall execution time taken by the alternatives. Finally, in the end, we conclude that we have successfully simulated a heterogeneous cloud environment in which we discarded the assumption that all the processing entities inside a host are on the same MIPS rating and have successfully allocated processing entities with different MIPS rating to virtual machines which are need of processing entities. The RISE-ORP allocates the virtual machines to the alternatives successfully. We have plotted the total number of alternatives executed successfully and have seen that the RISE-ORP framework surpasses the existing approach in terms of the total number of alternatives successfully executed.

Improving the RISE-ORP framework in order to gain less execution time is considered as the future work of this study. The RISE-ORP framework included optimization in it so as to reduce the global execution time. This study considered a single parameter for the optimization of the AHP algorithm for the resource provisioning. The optimization of AHP algorithm is now to be studied so as to include the other network based parameters for various resources and the Datacenters. The convergence speed of the RISE-ORP algorithm is also our further research direction.

\section{REFERENCES}

1. Rajkumar Buyya, Chee Shin Yeo, Srikumar Venugopal, James Broberg, and Ivona Brandic, 2009. "Cloud computing and emerging IT platforms: Vision, hype, and reality for delivering computing as the 5th utility" Future Generation computer systems,
25.6, 599-616, Elsevier.

2. Glauco Estácio Gonçalves, Patrícia Takako Endo, Thiago Damasceno Cordeiro, André Vitor de Almeida Palhares, Djamel Sadok, Judith Kelner, Bob Melander, and Jan-Erik Mångs, 2011 "Resource allocation in clouds: 
concepts, tools and research challenges." 22nd Brazilian Symposium on Computer Networks and Distributed Systems (SBRC), SBC.

3. Thomas Saaty, 1980 "The Analytic Hierarchy Process: Planning, Priority Setting, Resource, Allocation" McGraw-Hill, New York.

4. Thomas Saaty and Thomas L, 1990 "How to make a decision: the analytic hierarchy process. European journal of operational research", 48.1, 9-26, Elsevier.

5. Daji Ergu, Gang Kou, Yi Peng, Yong Shi, and Yu Shi, 2013 "The analytic hierarchy process: task scheduling and resource allocation in cloud computing environment." The Journal of Supercomputing, 64.3, 835848, Elsevier.

6. Xia-yu Hua, Jun Zheng, and Wen-xin Hu, 2010 "Ant colony optimization algorithm for computing resource allocation based on cloud computing environment" Journal of East China Normal University (Natural Science), 1, 127-134, Oriprobe.

7. Hu Wenxin Hu, Wen Xin, Jun Zheng, Xia Yu Hua, and Ya Qian Yang, 2013 "A Computing Capability Allocation Algorithm For Cloud Computing Environment." Applied Mechanics and Materials, 347, 2400-2406, Elsevier.

8. Zhe Gao, 2014 "The Allocation of Cloud Computing Resources Based on the Improved Ant Colony Algorithm" 2014 Sixth International Conference on Intelligent Human-Machine Systems and Cybernetics (IHMSC), 2, 334-337, IEEE.

9. Rodrigo N. Calheiros, Rajiv Ranjan, Anton Beloglazov, César AF De Rose, and Rajkumar Buyya, 2011 "CloudSim: a toolkit for modeling and simulation of cloud computing environments and evaluation of resource provisioning algorithms." Journal of Software: Practice and Experience, 41.1, 23-50, Wiley.

10. NR Ram Mohan, and E. Babu Raj, 2012 "Resource Allocation Techniques in Cloud Computing--Research Challenges for Applications", 2012 Fourth International Conference on Computational Intelligence and Communication Networks (CICN), 556560, IEEE.
11. Mohamed Abu Sharkh, Manar Jammal, Abdallah Shami, and Abdelkader Ouda, 2013 "Resource allocation in a networkbased cloud computing environment: design challenges". Communications Magazine, IEEE, 51.11, 46-52, IEEE.

12. V. Vinothina, R. Sridaran, and Padmavathi Ganapathi, 2012 "A survey on resource allocation strategies in cloud computing." International Journal of Advanced Computer Science and Applications 3.3, 97-104, SAI.

13. Shu, Wanneng, Wei Wang, and Yunji Wang, 2014 "A novel energy-efficient resource allocation algorithm based on immune clonal optimization for green cloud computing" .EURASIP Journal on Wireless Communications and Networking. arXiv preprint arXiv:1405.4618.

14. Xavier LeóN, and Leandro Navarro, 2013 "A Stackelberg game to derive the limits of energy savings for the allocation of data center resources." Future Generation Computer Systems 29.1, 74-83, Elsevier.

15. Maha Jebalia, A. Ben Letai"fa, Mohamed Hamdi, and Sami Tabbane, 2013 "A comparative study on game theoretic approaches for resource allocation in cloud computing architectures." 2013 IEEE 22nd International Workshop on Enabling Technologies: Infrastructure for Collaborative Enterprises (WETICE), 336-341, IEEE.

16. Iyer, Ganesh Neelakanta, and Bharadwaj Veeravalli, 2011 "On the resource allocation and pricing strategies in compute clouds using bargaining approaches". 2011 17th IEEE International Conference onNetworks (ICON), 147-152.

17. Bhat, Ramesh, Bharat Bhushan Verma, and Elan Reuben, 2001 " Data envelopment analysis (DEA)", Journal of Health Management, 3(2), 309-328.

18. K. Reddy S, and Ch. S. Rao, 2012 "Dynamic Resource Allocation In The Cloud Computing Using Nephele's Architecture", International Journal Of Engineering Science \& Advanced Technology(IJESAT), 2.4, $1144-1151$.

19. K. C. Gouda, Radhika, T. V., and Akshatha, M, 2013. "Priority based resource allocation model for cloud computing." International Journal of Science, Engineering and 
Technology Research (IJSETR), 22787798.

20. Gunho Lee, Niraj Tolia, Parthasarathy Ranganathan, \& Randy H. Katz. 2010 "Topology-aware resource allocation for data-intensive workloads." In Proceedings of the first ACM Asia-pacific workshop on Workshop on systems, 1-6, ACM.

21. Anil Singh, Kamlesh Dutta, and Avtar
Singh, 2014 "Resource Allocation in Cloud Computing Environment using AHP Technique". International Journal of Cloud Applications and Computing (IJCAC), 4.1, 33-44, IGI Global.

22. C. S. M. I. C. (CSMIC), "SMI Framework," URL http://betawww.cloudcommons.com/ servicemeasurementindex. 